\title{
Chemical Composition of the Essential Oil and Antimicrobial Activity of Scaligeria DC. Taxa and Implications for Taxonomy
}

\author{
Ayşe Baldemir $\odot^{1^{*}}$, Betül Demirci $\odot^{2}$, Mehmet Yavuz Paksoy $\odot^{3}$, \\ Selen İlgün $\odot^{1}$, Müberra Koşar $\odot^{4}$, Kemal Hüsnü Can Başer $\odot^{5}$ \\ and Fatih Demirci $\odot^{2,6}$ \\ ${ }^{I}$ Erciyes University, Faculty of Pharmacy, Department of Pharmaceutical Botany, \\ 38039, Kayseri, Türkiye \\ ${ }^{2}$ Anadolu University, Faculty of Pharmacy, Department of Pharmacognosy, 26470, Eskişehir, Türkiye \\ ${ }^{3}$ Tunceli University, Faculty of Engineering, Department of Environmental Engineering, \\ Tunceli, Türkiye \\ ${ }^{4}$ Eastern Mediterranean University, Faculty of Pharmacy, \\ Department of Pharmacognosy, L, K.K.T.C. \\ ${ }^{5}$ Near East University, Faculty of Pharmacy, Department of Pharmacognosy, 99138, \\ Nicosia, K.K.T.C. \\ ${ }^{6}$ Anadolu University, Faculty of Health Sciences 26470, Eskişehir, Türkiye
}

(Received February 22, 2017; Revised July 5, 2017; Accepted July 7, 2017)

\begin{abstract}
Six different Scaligeria DC. taxa (Apiaceae) essential oils (EOs) obtained by hydrodistillation from herba with the flowers collected from different sites from Turkey. The oils were analyzed and characterized by gas chromatography-flame ionization detector (GC-FID) and gas chromatography-mass spectrometry (GC-MS) simultaneously. A total of 133 different compounds were identified and relative qualitative and quantitative differences were observed among the evaluated samples. Analytical profiles of the Scaligeria EOs showed characteristic differences in terms of different main chemical constituents, between the two taxa $S$. lazica Boiss. and S. tripartita (Kalen.) Tamamsch; and S. napiformis (Sprengel) Grande, S. meifolia (Fenzl) Boiss., S. capillifolia Post, S. hermonis Post, S. glaucescens (DC.) Boiss. taxa, respectively. The main component germacrene D can be utilized as marker for the chemical discrimination of the Scaligeria genus. In addition, Scaligeria EOs were evaluated in vitro for their antimicrobial activity against pathogenic Gram positive (Staphylococcus aureus and Bacillus cereus), Gram negative (Pseudomonas aeruginosa) and yeast (Candida albicans, C. parapsilosis, and C. krusei) standard strains by using a micro-dilution assay. As a general result, the oils showed moderate inhibitory range when compared with standard antimicrobial agents.
\end{abstract}

Keywords: Scaligeria sp.; chemo-taxonomy; essential oil; antimicrobial activity, Turkey. (C) 2017 ACG Publications. All rights reserved.

\section{Introduction}

Aromatic plants are often used in traditional medicine for their antimicrobial activities due to their essential oils (EOs), volatile compounds since ancient times. Several components such as

\footnotetext{
* Corresponding author: E-Mail: aysebaldemir@gmail.com; Phone: +90-352-2076666 Fax: +90-352-4379169. 
lipophilic compounds including terpenoid derivatives of a variety of essential oils showed significant in vitro antimicrobial activity against bacteria, yeasts, dermatophyte, and fungal strains such as Aspergillus [1-4], and also have therapeutic potential in different infectious diseases. When elaborating the mode of action, the components in essential oils have been demonstrated to disrupt cellular membranes in bacteria and fungi, hence inhibiting cellular respiration and ionic transport [58].

The Apiaceae family products are among the prominent EOs, which are rich in terpenoids and phenylpropanoids [8-13]. This family is represented by 101 genera comprising 485 species of which 181 are endemic species in Turkey [14]. Scaligeria DC. genus is represented by 7 species of which two are endemic in Turkey. The species recorded in Turkish Flora are listed as follows: S. napiformis (Sprengel) Grande, S. tripartita (Kalen.) Tamamsch, S. lazica Boiss. (endemic), S. meifolia (Fenzl) Boiss., S. glaucescens (DC.) Boiss., S. hermonis Post, S. capillifolia Post (endemic) $[15,16]$. Stevens stated that Scaligeria DC. spp. is an annual (which is not clear), biennial and perennial plant with white flowers and smooth fruit with globose shape [15]. Historically and traditionally Scaligera species from Turkey have been associated with anise, and their local names were given inspired from this similarity both from the morphology and aromatic characteristics. Thus, Scaligeria species are generally named using "K1l anason", "Puslu anason", "Laz anasonu", "Uzun anason" etc. referring to anise [16].

Previous studies and phytochemical investigations showed that the chemical composition profiles of Scaligeria and Pimpinella species are surprisingly similar [17]. For the initial characteristic phytochemical discrimination of Scaligeria genus from Turkey, the chemical composition and antimicrobial effects of EOs from 6 taxa were evaluated comparatively in this present study, to the best of our knowledge for the first time.

\section{Materials and Methods}

\subsection{Plant Materials}

The herbal parts of Scaligeria species were collected in the flowering period (2014-2016) from different regions of Turkey. The plant specimens were deposited in the Science Faculty of Erciyes University, Kayseri-Turkey. In this regard, the voucher numbers of the studied species are listed in Table 1.

\subsection{Isolation of the Essential Oil}

The plant materials were air dried at room temperature and were subjected to hydrodistillation for $3 \mathrm{hrs}$ using a Clevenger-type apparatus to extract essential oils. The percentage yields were calculated on a dry weight basis as given in Table 1 . The oils were dried over anhydrous sodium sulfate to remove moisture and stored at $+4{ }^{\circ} \mathrm{C}$ until analyzed and tested further.

\subsection{Analysis of Essential Oil}

\subsubsection{GC-FID Condition}

The GC analysis was carried out using an Agilent $6890 \mathrm{~N}$ GC system. Innowax FSC column (60 $\mathrm{m} \times 0.25 \mathrm{~mm}, 0.25 \mu \mathrm{m}$ film thickness) was used as described for the GC-MS. FID detector temperature was $300^{\circ} \mathrm{C}$. To obtain the same elution order with the GC-MS system, simultaneous autoinjection was performed on a duplicate of the same column applying the same operational conditions. Relative percentage amounts (\%) of the separated compounds were calculated from FID chromatograms. The analysis results are given in Table 2.

The individual essential oil components were identified by comparison of their relative retention times with those of th authentic samples or by comparison of their relative retention index 
(RRI) to series of $n$-alkanes. Computer matching against commercial (Wiley GC/MS Library, MassFinder3 Library) $[18,19]$ and in-house "Başer Library of Essential Oil Constituents" built up by genuine compounds and components of known oils, as well as MS literature data [20,21], was used for the identification.

\subsubsection{GC-MS Condition}

The analyses were carried out using an Agilent 5975 GC-MSD system. Innowax FSC column ( $60 \mathrm{~m} \times 0.25 \mathrm{~mm}, 0.25 \mu \mathrm{m}$ film thickness) was used with helium as carrier gas $(0.8 \mathrm{~mL} / \mathrm{min})$. GC oven temperature was kept at $60{ }^{\circ} \mathrm{C}$ for $10 \mathrm{~min}$ and programmed to $220{ }^{\circ} \mathrm{C}$ at a rate of $4{ }^{\circ} \mathrm{C} / \mathrm{min}$, and kept constant at $220{ }^{\circ} \mathrm{C}$ for $10 \mathrm{~min}$ and then programmed to $240{ }^{\circ} \mathrm{C}$ at a rate of $1{ }^{\circ} \mathrm{C} / \mathrm{min}$. Split ratio was adjusted at 40:1. The injector temperature was set at $250^{\circ} \mathrm{C}$. Mass spectra were recorded at $70 \mathrm{eV}$. Mass range was from $m / z 35$ to 450 .

Table 1. Scaligeria species collection sites, yields and codes.

\begin{tabular}{|c|c|c|c|c|c|}
\hline Species & Collection site & Code & $\begin{array}{l}\text { Collection } \\
\text { date }\end{array}$ & $\begin{array}{l}\text { Herbarium } \\
\text { number }\end{array}$ & $\begin{array}{c}\text { Oil } \\
\text { yield* } \\
(\%)\end{array}$ \\
\hline S. lazica & Artvin: Borçka, Maral forest, roadside & SL-1 & July 2014 & ERCH 5101 & 0.19 \\
\hline S. lazica & $\begin{array}{l}\text { Trabzon: Köprübaşı, Beşköy, } \\
\text { Büyükdoğanlı village } 800 \mathrm{~m} .\end{array}$ & SL-2 & July 2014 & ERCH 5102 & 0.08 \\
\hline S. lazica & $\begin{array}{l}\text { Trabzon: Altındere, Sümela monastery } \\
\text { surroundings }\end{array}$ & SL-3 & July 2014 & ERCH 5103 & 0.38 \\
\hline S. napiformis ${ }^{\mathrm{a}}$ & $\begin{array}{l}\text { Antalya: Akseki, Çukur village, Istarlas } \\
\text { located, } 1050 \mathrm{~m} \text {. }\end{array}$ & SN-1 & May 2014 & ERCH 5104 & 0.10 \\
\hline S. napiformis ${ }^{\mathrm{b}}$ & $\begin{array}{l}\text { Muğla: Köyceğiz, Ekincik village, } \\
\text { Sandallıtepe located, } 480 \mathrm{~m} \text {. }\end{array}$ & SN-2 & May 2014 & ERCH 5105 & 0.10 \\
\hline S. meifolia & $\begin{array}{l}\text { Siirt: Pervari, between Tandırköy and } \\
\text { Göl village gateway, in among oaks }\end{array}$ & SM-1 & June 2014 & ERCH 5106 & 0.04 \\
\hline S. meifolia & $\begin{array}{l}\text { Mardin: Bakırkırı, Şeyhşaran tomb } \\
\text { surroundings, in among oaks, } 1020 \mathrm{~m} \text {. }\end{array}$ & SM-2 & June 2014 & ERCH 5107 & 0.01 \\
\hline S. capillifolia & $\begin{array}{l}\text { Osmaniye: South of Yarpuz, Yağlıpınar } \\
\text { located, } 1400 \mathrm{~m} \text {. }\end{array}$ & SC-1 & July 2014 & ERCH 5108 & 0.18 \\
\hline S. tripartita & $\begin{array}{l}\text { Rize: Çamlıhemşin, between Çat and } \\
\text { Orta village, } 1750 \mathrm{~m} \text {. }\end{array}$ & ST-1 & June 2016 & ERCH 5109 & 0.05 \\
\hline S. glaucescens & $\begin{array}{l}\text { Van: between Van and Muradiye, } 7 \mathrm{~km} \\
\text { before Karahan, steppe slopes, } 1685 \mathrm{~m} \text {. }\end{array}$ & SG-1 & June 2016 & ERCH 5110 & 0.10 \\
\hline S. hermonis & $\begin{array}{l}\text { Konya: between Seydişehir and içeri } \\
\text { kışla, } 1200 \mathrm{~m} \text {. }\end{array}$ & SH-1 & June 2016 & ERCH 5111 & 0.05 \\
\hline
\end{tabular}

"Calculated on moisture-free basis.

${ }^{\mathrm{a}, \mathrm{b}}$ Essential oil components of S. napiformis were reported by Baldemir et al. (2016) [22].

\subsection{In vitro Antimicrobial Evaluation}

In vitro antimicrobial activity of the Scaligeria sp. EOs (SC-1, SL-1, SL-2, SL-3, SH-1, ST-1) were evaluated using the microdilution method according to Clinical and Laboratory Standards institute (CLSI) guidelines [23, 24]. Chloramphenicol, Ciprofloxacine, Amphotericin B and Ketoconazole were used as standard antimicrobial agents, where applicable. All experiments were repeated in triplicate and average MIC results are listed in Table 3. 


\section{Results and Discussion}

The initial EO yields of Scaligeria species (S. tripartita, S. lazica, S. meifolia, S. glaucescens, S. hermonis, $S$. capillifolia) were variable and ranged from 0.01 to $0.38 \%$, collected from different regions of the Turkey (Table 1). To the best our knowledge, this is the first report on the comparative study of Scaligeria taxa and their subsequent essential oil chemical compositions.

EOs were analyzed both by GC-FID and GC-MS using a polar column, and constituents were compared to known compounds using the in-house Baser Library and Wiley GC/MS Library, MassFinder3 Library. As a result; one hundred and thirty three different compounds were identified in Scaligeria EOs, which constituted 59.7-100\% of the total oil. These compounds are listed in Table 2 with their relative percentages.

The use and utilization of EOs bearing plants in traditional medicine systems is being practiced since ancient times in human history [33]. Aerial parts of S. cretica (Mill.) Boiss. is used for splenomegaly and for jaundice in the Southwest Balkan, Peninsula and Asia Minor [34]. The Scaligeria species essential oil composition, various biological activity studies were reported in previous studies [17, 22, 35-39]. However, comprehensive studies on the Scaligeria genus are still very limited and not enough to clarify their existing taxonomic problems. In previous studies, some chemical components characteristic to Pimpinella spp. were also detected in S. tripartita and S. lazica species. For instance, S. tripartita fruit oil was rich in geijerenes and its root oil contained epoxypseudoisoeugenylangelate and geijerene; which were identified as antibacterial compounds by bioautography method [17]. It is well known that $\mathrm{C} 12$ sesquiterpenes (geijerene, pregeijerene) and phenylpropanoids (pseudoisoeugenyl 2-methylbutyrate, epoxypseudoisoeugenyl 2-methylbutyrate) are phytochemical markers for the Pimpinella genera [40-42]. In another study, while fruit oil of Scaligeria lazica was found as a rich source of $(\mathrm{Z})-\beta$-farnesene $(89,2 \%)$, the oil of the aerial parts was rich in $(E)$-anethole $(49,7 \%)$. In this present study, these substances were determined in SL-1, SL-2 and SL-3 oils. Also, geijerene, pregeijerene B, isogeijerene, cogeijerene known as C12 sesquiterpenes could be characterized in ST-1 oil, especially geijerene and pregeijerene B were the main constituents (Figure 1). Interestingly, geijerenes were previously detected in different families such as Asteraceae, Lamiaceae, Rosaceae, Rutaceae and Fabaceae. However, the geijerenes were present only in the Pimpinella genus of the Apiaceae family [41, 42-46]. The stems and leaves, fruit and root essential oil components of $S$. tripartita confirmed our results that ST-1 oil contained $36.8 \%, 54.6 \%$ and $28.5 \%$ geijerene, respectively [17]. Furthermore, $(E)$-anethole was identified as the main component in $S$. lazica essential oil of the aerial parts both in our study and in previously reported studies $[35,36]$. Rowshan and Tarakemeh (2013) reported that main constituents of S. meifolia essential oil of the aerial parts collected from Iran were germacrene D, germacrene B and limonene. In our study, while $\alpha$-copaene, cubebol, hexadecanoic acid were the major components for SM-1 oil, caryophyllene oxide, hexadecanoic acid, $\alpha$-copaene and cubebol are components were the main components for the SM-2 oil, respectively (Table 3). However, the main components identified in S. meifolia collected from two different localities in our work, were different from the components reported in a previous work [39]. 
Table 2. Relative percentages (\%) of the essential oils of herb with flowering of Scaligeria species gathered from Turkey.

\begin{tabular}{|c|c|c|c|c|c|c|c|c|c|c|c|}
\hline $\mathbf{K I}^{\mathbf{a}}$ & $\mathbf{R R I}^{\mathrm{b}}$ & Compound & SH-1 & SC-1 & SL-1 & SL-2 & SM-1 & ST-1 & SL-3 & SM-2 & SG-1 \\
\hline $1025^{\mathrm{c}}$ & 1032 & $\alpha$-Pinene & - & - & - & - & - & $\mathrm{t}$ & - & - & 2.3 \\
\hline $1117^{\mathrm{c}}$ & 1118 & $\beta$-Pinene & - & 0.5 & - & - & - & - & - & - & 4.0 \\
\hline $1122^{c}$ & 1132 & Sabinene & - & - & - & - & - & - & - & - & 0.1 \\
\hline $1146^{\mathrm{d}}$ & 1159 & $\delta$-3-Carene & - & - & - & 0.3 & - & - & - & - & - \\
\hline $1160^{\mathrm{c}}$ & 1174 & Myrcene & - & - & - & - & - & - & - & - & 0.6 \\
\hline $118^{\mathrm{d}}$ & 1194 & Heptanal & - & - & - & - & 4.9 & - & - & 2.2 & 1.1 \\
\hline $1212^{\mathrm{c}}$ & 1203 & Limonene & - & - & - & - & - & - & - & - & 1.0 \\
\hline $1209^{c}$ & 1218 & $\beta$-Phellandrene & - & - & - & - & - & - & - & - & 2.2 \\
\hline $1232^{\mathrm{d}}$ & 1244 & 2-Pentyl furan & - & - & - & - & - & - & - & - & $\mathrm{t}$ \\
\hline $1232^{\mathrm{c}}$ & 1246 & (Z)-B-Ocimene & $\mathrm{t}$ & - & - & - & - & - & - & - & 4.5 \\
\hline $1245^{\mathrm{c}}$ & 1255 & $\gamma$-Terpinene & - & - & - & - & - & - & - & - & 0.4 \\
\hline $1249^{c}$ & 1266 & $(E)-\beta$-Ocimene & 0.1 & - & - & - & - & - & - & - & 1.8 \\
\hline $1282^{\mathrm{c}}$ & 1280 & $p$-Cymene & - & - & - & - & - & $\mathrm{t}$ & - & - & 1.0 \\
\hline $1282^{\mathrm{c}}$ & 1290 & Terpinolene & - & - & - & - & - & - & - & - & 0.3 \\
\hline \multirow[t]{2}{*}{$1287^{\mathrm{d}}$} & 1296 & Octanal & 0.3 & - & - & - & - & - & - & - & 0.3 \\
\hline & 1337 & Geijerene & 0.1 & - & - & - & - & 12.4 & - & - & - \\
\hline $1391^{\mathrm{d}}$ & 1400 & Nonanal & $\mathrm{t}$ & - & - & - & - & - & - & - & 0.2 \\
\hline \multirow[t]{3}{*}{$1480^{c}$} & 1466 & $\alpha$-Cubebene & 0.1 & - & - & - & 1.0 & - & - & 1.2 & 0.7 \\
\hline & 1490 & Isogeijerene & - & - & - & - & - & 0.3 & - & - & - \\
\hline & 1492 & Cyclosativene & - & - & - & - & - & - & - & - & 0.4 \\
\hline $1488^{\mathrm{c}}$ & 1497 & $\alpha$-Copaene & 0.7 & 3.8 & - & 0.3 & 7.2 & 0.1 & - & 5.2 & 12.0 \\
\hline $1495^{\mathrm{d}}$ & 1506 & Decanal & - & - & - & - & - & - & - & - & 0.2 \\
\hline \multirow[t]{2}{*}{$1523^{c}$} & 1535 & $\beta$-Bourbonene & 0.3 & - & - & - & - & - & - & - & - \\
\hline & 1544 & Cogeijerene* & - & - & - & - & - & 1.0 & - & - & - \\
\hline
\end{tabular}


Table 2. (Continued).

\begin{tabular}{|c|c|c|c|c|c|c|c|c|c|c|c|}
\hline $\mathbf{K I}^{\mathrm{a}}$ & $\mathbf{R R I}^{\mathrm{b}}$ & Compound & SH-1 & SC-1 & SL-1 & SL-2 & SM-1 & ST-1 & SL-3 & SM-2 & SG-1 \\
\hline $1541^{\mathrm{d}}$ & 1549 & $\beta$-Cubebene & 0.8 & 0.2 & - & - & - & - & - & - & 5.2 \\
\hline $1543^{c}$ & 1553 & Linalool & 0.3 & - & - & - & - & 0.2 & 0.4 & - & - \\
\hline \multirow[t]{2}{*}{$1575^{\mathrm{d}}$} & 1568 & trans- $\alpha$-Bergometene & - & - & - & - & - & - & - & - & - \\
\hline & 1572 & Pregeijerene B & - & - & - & - & - & 59.2 & - & - & - \\
\hline $1576^{\mathrm{c}}$ & 1589 & $\beta$-Ylangene & 0.2 & - & - & - & - & - & - & - & 0.3 \\
\hline \multirow[t]{3}{*}{$1579^{c}$} & 1591 & Bornylacetate & - & 0.9 & - & - & - & - & - & - & 0.7 \\
\hline & 1594 & trans- $\beta$-Bergamotene & - & 0.2 & - & - & - & - & 0.1 & - & - \\
\hline & 1595 & Isothymol methyl ether & - & - & - & - & - & - & - & - & 0.1 \\
\hline $1579^{c}$ & 1597 & $\beta$-Copaene & 0.2 & - & - & - & - & - & - & - & \\
\hline \multirow[t]{2}{*}{$1590^{\mathrm{c}}$} & 1600 & $\beta$-Elemene & 0.2 & - & - & - & - & 0.1 & - & - & 0.1 \\
\hline & 1604 & Thymol methyl ether & - & - & - & - & - & - & - & - & 0.2 \\
\hline \multirow[t]{3}{*}{$1608^{c}$} & 1612 & $\beta$-Caryophyllene & 0.9 & 1.6 & 24.9 & 12.9 & - & 0.8 & 17.8 & - & 0.9 \\
\hline & 1613 & Acora-2,4-diene & - & 1.6 & - & - & - & - & - & - & - \\
\hline & 1617 & 6,9-Guaiadiene & - & - & - & - & - & - & 0.3 & - & - \\
\hline $1639^{\mathrm{d}}$ & 1655 & (E)-2-Decenal & - & - & - & - & 1.8 & - & - & 1.4 & 0.4 \\
\hline $1651^{\mathrm{d}}$ & 1668 & (Z)- $\beta$-Farnesene & - & - & 4.0 & 4.1 & 1.0 & 3.8 & 4.8 & 2.9 & - \\
\hline $1661^{\mathrm{c}}$ & 1670 & trans-Pinocarveol & - & - & - & - & - & - & - & - & - \\
\hline $1663^{c}$ & 1687 & $\alpha$-Humulene & 0.2 & 0.5 & 1.8 & 0.9 & - & - & 1.2 & - & - \\
\hline $1687^{\mathrm{d}}$ & 1693 & $\beta$-Acoradiene & 0.6 & - & - & - & - & - & - & - & - \\
\hline $1689^{c}$ & 1704 & $\gamma$-Muurolene & 0.2 & 1.1 & - & - & - & - & - & - & 0.4 \\
\hline
\end{tabular}


Table 2. (Continued).

\begin{tabular}{|c|c|c|c|c|c|c|c|c|c|c|c|}
\hline $\mathbf{K I}^{\mathbf{a}}$ & $\mathbf{R R I}^{\mathrm{b}}$ & Compound & SH-1 & SC-1 & SL-1 & SL-2 & SM-1 & ST-1 & SL-3 & SM-2 & SG-1 \\
\hline \multirow{3}{*}{$1708^{d}$} & 1707 & trans-Muurola-4(14),5-diene* & - & 4.6 & - & - & - & - & - & - & - \\
\hline & 1711 & $\gamma$-Himachalene & - & - & - & 0.5 & - & - & - & - & - \\
\hline & 1718 & 4,6-Guaiadine & - & 0.4 & - & - & - & - & - & - & - \\
\hline $1711^{\mathrm{d}}$ & 1722 & Dodecanal & - & - & - & - & - & - & - & - & 0.1 \\
\hline $1708^{c}$ & 1726 & Germacrene D & 58.9 & - & 1.6 & 1.1 & - & - & 1.2 & - & 18.7 \\
\hline $1720^{\mathrm{d}}$ & 1726 & $\alpha$-Zingiberene & - & - & 1.9 & 1.1 & - & - & 2.1 & - & - \\
\hline $1723^{c}$ & 1740 & $\alpha$-Muurolene & - & - & - & - & - & - & - & - & 0.6 \\
\hline \multirow[t]{2}{*}{$1734^{\mathrm{d}}$} & 1741 & $\beta$-Bisabolene & - & - & - & 0.6 & - & - & 0.9 & - & - \\
\hline & 1742 & $\beta$-Selinene & - & 3.4 & - & - & - & - & - & - & - \\
\hline $1734^{\mathrm{c}}$ & 1755 & Bicyclogermacrene & 2.5 & - & - & - & - & 0.7 & - & - & 0.7 \\
\hline $1754^{\mathrm{d}}$ & 1766 & 1-Decanol & - & - & - & - & - & - & - & - & 0.3 \\
\hline $1755^{\mathrm{c}}$ & 1773 & $\delta$-Cadinene & 0.5 & 23.0 & 1.0 & 0.3 & 1.4 & - & - & 3.5 & 1.9 \\
\hline $1763^{c}$ & 1776 & $\gamma$-Cadinene & 0.1 & - & - & - & - & - & - & - & - \\
\hline $1781^{\mathrm{c}}$ & 1786 & ar-Curcumene & - & - & 3.1 & 1.8 & 1.0 & - & 2.3 & 1.1 & - \\
\hline $1808^{d}$ & 1827 & $(E, E)-2,4-$ Decadienal & - & - & - & - & - & - & - & - & 0.3 \\
\hline \multirow[t]{6}{*}{$1826^{d}$} & 1845 & (E)-Anethol & - & - & 43.0 & 49.4 & - & 0.3 & 38.0 & - & - \\
\hline & 1853 & cis-Calamenene & 0.1 & 0.4 & - & - & 1.6 & - & - & 1.1 & - \\
\hline & 1868 & $(E)$-Geranyl acetone & - & - & - & - & $\mathrm{t}$ & - & - & 1.4 & - \\
\hline & 1870 & trans-Calamenene & - & 0.3 & - & - & - & - & - & - & - \\
\hline & 1871 & Traginone & - & - & - & - & - & 1.0 & - & - & - \\
\hline & 1898 & 1,11-Oxidocalamenene & - & 0.1 & - & - & - & - & - & - & - \\
\hline
\end{tabular}


Table 2. (Continued).

\begin{tabular}{|c|c|c|c|c|c|c|c|c|c|c|c|}
\hline $\mathbf{K I}^{\mathbf{a}}$ & RRI $^{\text {b }}$ & Compound & SH-1 & SC-1 & SL-1 & SL-2 & SM-1 & ST-1 & SL-3 & SM-2 & SG-1 \\
\hline $1900^{c}$ & 1900 & epi-Cubebol & 0.1 & - & - & - & 6.2 & - & - & 4.5 & 1.2 \\
\hline $1926^{\mathrm{d}}$ & 1933 & Tetradecanal & - & - & - & - & - & - & - & - & 0.2 \\
\hline \multirow[t]{3}{*}{$1921^{\mathrm{d}}$} & 1941 & $\alpha$-Calacorene & - & 3.5 & - & - & - & - & - & - & 0.2 \\
\hline & 1942 & $\begin{array}{l}\text { 4-Hydroxy-2-methyl } \\
\text { acetophenone }\end{array}$ & - & - & - & 0.5 & - & - & 0.3 & - & - \\
\hline & 1945 & 1,5-Epoxy-salvial(4)14-ene & 0.7 & - & - & - & - & - & - & - & 0.3 \\
\hline $1941^{\mathrm{d}}$ & 1957 & Cubebol & 0.1 & - & - & - & 7.4 & - & - & 5.2 & 1.0 \\
\hline $1935^{\mathrm{d}}$ & 1958 & (E)- $\beta$-Ionone & - & - & - & - & - & - & - & - & 0.3 \\
\hline $1959^{\mathrm{d}}$ & 1973 & 1-Dodecanol & - & - & - & - & $\mathrm{t}$ & - & - & 1.1 & 0.4 \\
\hline $1960^{\mathrm{e}}$ & 1984 & $\gamma$-Calacorene & - & 1.2 & - & - & - & - & - & - & - \\
\hline $2001^{\mathrm{f}}$ & 2001 & Isocaryophyllene oxide & - & - & $\mathrm{t}$ & 1.0 & - & - & 0.5 & - & - \\
\hline $1986^{\mathrm{d}}$ & 2008 & Caryophyllene oxide & 0.9 & 2.6 & 9.8 & 7.7 & 11.2 & 0.1 & 3.0 & 9.8 & 1.9 \\
\hline $2016^{c}$ & 2037 & Salvial-4(14)-en-1-one & 1.9 & - & - & - & 3.4 & - & - & 3.1 & 1.5 \\
\hline $2010^{\mathrm{c}}$ & 2053 & Anisaldehyde & - & - & - & 0.3 & - & - & 0.4 & - & - \\
\hline $2047^{\mathrm{c}}$ & 2071 & Humulene epoxide-II & - & 0.6 & - & - & - & - & $\operatorname{tr}$ & 1.7 & 0.2 \\
\hline $2067^{d}$ & 2080 & Cubenol & - & - & - & - & 0.9 & - & - & 1.2 & - \\
\hline $2057^{\mathrm{d}}$ & 2084 & Octanoic acid & - & - & - & - & 1.2 & - & - & - & - \\
\hline $2088^{c}$ & 2088 & 1-epi-Cubenol & - & - & - & - & 2.2 & - & - & 2.8 & 0.4 \\
\hline $2074^{d}$ & 2095 & Hexyl benzoate & - & - & - & - & 4.1 & - & - & 3.7 & - \\
\hline $2078^{d}$ & 2096 & Elemol & - & - & - & - & - & 0.1 & - & - & - \\
\hline $2130^{\mathrm{g}}$ & 2130 & Salviadienol & 0.8 & - & - & - & - & - & - & - & 0.7 \\
\hline $2124^{\mathrm{c}}$ & 2131 & Hexahydrofarnesyl acetone & - & - & - & - & 1.3 & - & - & 2.3 & 0.2 \\
\hline $2144^{\mathrm{h}}$ & 2143 & Rosifoliol & - & 0.4 & - & - & - & - & - & - & - \\
\hline $2126^{\mathrm{c}}$ & 2144 & Spathulenol & 2.9 & 0.2 & $\mathrm{t}$ & 0.9 & 3.8 & 0.1 & 0.5 & 2.5 & 1.9 \\
\hline
\end{tabular}


Table 2. (Continued).

\begin{tabular}{|c|c|c|c|c|c|c|c|c|c|c|c|}
\hline $\mathbf{K I}^{\mathbf{a}}$ & $\mathbf{R R I}^{\mathbf{b}}$ & Compound & SH-1 & SC-1 & SL-1 & SL-2 & SM-1 & ST-1 & SL-3 & SM-2 & SG-1 \\
\hline \multirow[t]{9}{*}{$2119^{d}$} & 2148 & (Z)-3-Hexen-1-yl benzoate & 0.3 & - & - & - & - & - & - & - & - \\
\hline & 2148 & Dictamnol & - & - & - & - & - & 1.4 & - & - & - \\
\hline & 2113 & Guaiyl acetate & - & 2.0 & - & - & - & - & - & - & - \\
\hline & 2161 & Muurola-4,10(14)-dien-1-ol & - & 2.3 & - & - & 1.4 & - & - & 2.3 & 0.8 \\
\hline & 2166 & Osmorhizol & - & - & - & - & - & - & - & - & - \\
\hline & 2179 & nor-Copaonone & 1.4 & - & - & - & - & - & - & - & - \\
\hline & 2179 & 1-Tetradecanol & - & - & - & - & 4.9 & - & - & 2.0 & 0.4 \\
\hline & 2180 & Junenol (=Eudesm-4(15)-en-6-ol) & 0.3 & - & - & - & - & - & - & - & - \\
\hline & 2180 & $\beta$-Copaen- $4 \alpha-\mathrm{ol}^{*}$ & - & - & - & - & 3.9 & - & - & 4.9 & - \\
\hline \multirow[t]{3}{*}{$2165^{\mathrm{c}}$} & 2187 & T-Cadinol & - & 0.3 & - & - & - & - & - & - & - \\
\hline & 2209 & T-Muurolol & 0.4 & 0.4 & - & - & - & - & - & - & - \\
\hline & 2214 & ar-Turmerol & - & - & - & 0.3 & - & - & 0.1 & 1.7 & - \\
\hline $2183^{d}$ & 2219 & $\delta$-Cadinol ( $=\alpha$-muurolol) & - & 0.1 & - & - & - & - & - & - & - \\
\hline $2227^{\mathrm{c}}$ & 2255 & $\alpha$-Cadinol & 0.5 & - & - & - & - & - & - & - & 0.3 \\
\hline $2233^{c}$ & 2256 & Cadalene & - & 1.0 & - & - & - & - & - & - & - \\
\hline \multirow[t]{2}{*}{$2252^{\mathrm{c}}$} & 2273 & Selin- $11-$ en- $4 \alpha-$ ol & - & 0.5 & - & - & - & - & - & - & - \\
\hline & 2278 & Torilenol & 1.4 & - & - & - & - & - & - & - & 2.0 \\
\hline $2300^{j}$ & 2300 & Tricosane & - & - & - & - & - & - & - & - & 0.2 \\
\hline $2316^{\mathrm{f}}$ & 2316 & $\begin{array}{l}\text { Caryophylla-2(12),6(13)-dien-5 } 3 \text { - } \\
\text { ol (=Caryophylladienol I) }\end{array}$ & - & - & - & 0.7 & - & - & 0.2 & 2.0 & - \\
\hline $2366^{\mathrm{c}}$ & 2369 & Eudesma-4(15),7-dien-1 $\beta$-ol & 2.1 & - & - & - & - & - & - & - & 2.0 \\
\hline $2374^{\mathrm{d}}$ & 2384 & 1-Hexadecanol & - & - & - & - & 2.3 & - & - & 2.2 & - \\
\hline
\end{tabular}


Table 2. (Continued).

\begin{tabular}{|c|c|c|c|c|c|c|c|c|c|c|c|}
\hline $\mathbf{K I}^{\mathbf{a}}$ & $\underset{b}{\mathbf{R R I}}$ & Compound & SH-1 & SC-1 & SL-1 & SL-2 & SM-1 & ST-1 & SL-3 & SM-2 & SG-1 \\
\hline \multirow[t]{2}{*}{$2392^{f}$} & 2392 & $\begin{array}{l}\text { Caryophylla-2(12),6-dien-5 } \beta \text {-ol } \\
\text { (=Caryophyllenol II) }\end{array}$ & - & 0.4 & - & 0.6 & - & - & 0.3 & - & - \\
\hline & 2411 & $\begin{array}{l}\text { 4-Isopropyl-6-methyl-1- } \\
\text { tetralone }\end{array}$ & - & 0.9 & - & - & - & - & - & - & - \\
\hline \multirow[t]{3}{*}{$2500^{\mathrm{f}}$} & 2500 & Pentacosane & - & - & - & - & 3.6 & - & - & 4.2 & - \\
\hline & 2568 & 14-Hydroxy- $\alpha$-muurolene & 0.4 & - & - & - & - & - & - & - & - \\
\hline & 2607 & 14-Hydroxy- $\delta$-cadinene & 0.7 & - & - & - & - & - & - & - & - \\
\hline \multirow[t]{2}{*}{$2613^{\mathrm{d}}$} & 2622 & Phytol & 0.5 & - & - & - & - & 0.1 & - & 1.9 & - \\
\hline & 2663 & (Z)-Pseudoisoeugenyltiglate & - & - & $\mathrm{t}$ & 0.9 & & 0.2 & 2.9 & & - \\
\hline \multirow[t]{5}{*}{$2500^{k}$} & 2700 & Heptacosane & - & - & - & - & 1.1 & - & - & 2.7 & - \\
\hline & 2766 & (E)-Pseudoisoeugenyltiglate & - & - & $\mathrm{t}$ & 0.7 & - & - & 0.8 & - & - \\
\hline & 2800 & $\begin{array}{l}\text { Epoxy-trans-pseudoisoeugenyl } \\
\text { angelate }\end{array}$ & - & - & 5.7 & 6.7 & - & 8.7 & 16.4 & - & - \\
\hline & 2887 & $\begin{array}{l}\text { Epoxy-trans-pseudoisoeugenyl } \\
\text { tiglate }\end{array}$ & - & - & 3.2 & 2.0 & - & - & 1.8 & - & - \\
\hline & 2900 & Nonacosane & - & 0.4 & - & - & 3.2 & - & - & 1.8 & 1.6 \\
\hline \multirow[t]{2}{*}{$2913^{c}$} & 2931 & Hexadecanoicacid & - & 0.3 & $\mathrm{t}$ & 1.3 & 7.1 & - & 0.7 & 5.3 & 3.6 \\
\hline & & Total identified (\%) & 82.7 & 59.7 & 100.0 & 96.9 & 89.1 & 90.6 & 97 & 88.9 & 85.3 \\
\hline
\end{tabular}

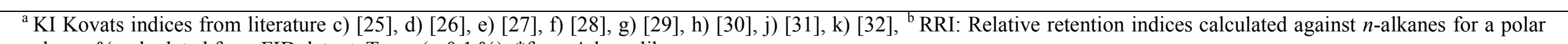
column, $\%$ calculated from FID data, t Trace $(<0.1 \%), *$ from Adams library 
Table 3. Antimicrobial activity results of Scaligeria essential oils (mg/mL).

\begin{tabular}{|c|c|c|c|c|c|c|}
\hline Test sample & $\begin{array}{c}\text { Bacillus } \\
\text { cereus } \\
\text { NRRL B- } \\
3711\end{array}$ & $\begin{array}{c}\text { Staphylococcus } \\
\text { aureus } \\
\text { ATCC } \\
\text { BAA } 1026\end{array}$ & $\begin{array}{c}\text { Pseudomonas } \\
\text { aeruginosa } \\
\text { ATCC } 10145\end{array}$ & $\begin{array}{c}\text { Candida } \\
\text { albicans } \\
\text { ATCC } \\
90028\end{array}$ & $\begin{array}{c}C . \\
\text { parapsilosis } \\
\text { ATCC } \\
22019\end{array}$ & $\begin{array}{c}C . \\
\text { krusei } \\
\text { ATCC } \\
6258\end{array}$ \\
\hline SC-1 & 0.32 & 0.32 & 0.64 & 0.64 & 0.64 & 0.64 \\
\hline SL-1 & 0.64 & 0.64 & 0.64 & 0.64 & 0.64 & 0.64 \\
\hline SL-2 & 0.64 & 0.64 & 0.64 & 0.64 & 0.64 & 0.64 \\
\hline SL-3 & 0.64 & 0.64 & 0.64 & 0.64 & 0.64 & 0.64 \\
\hline SH-1 & 0.32 & $>2.56$ & 2.56 & 2.56 & 2.56 & 2.56 \\
\hline ST-1 & 2.56 & $>8.96$ & $>8.96$ & 2.56 & 8.96 & 8.96 \\
\hline Amphotericin B & - & - & - & 8 & 64> & 64> \\
\hline Chloramphenicol & 8 & 32 & 8 & - & - & - \\
\hline Ketokonazole & - & - & - & 32 & 32 & 32 \\
\hline Ciprofloxacin & $2>$ & 16 & 2> & - & - & - \\
\hline
\end{tabular}

**The quantitative amounts of SN-1, SN-2, SM-1, SM-2 and SG-1 essential oil were insufficient.

The EOs of the leaf and fruit of S. assyriaca Freyn \& Bornm. were analysed. Germacrene D, $\beta$-Sesquiphellandrene and kessane were detected in both oil and identified ranging $(21.1 \%$ and $13.7 \%)$, $(10 \%$ and $8.7 \%)$ and $(7.4 \%$ and $9.5 \%)$ in leaf and fruit oils, respectively [37]. Main components of the EO of S. cretica (Miller) Boiss. are $\alpha$-pinene, $\beta$-farnesene and germacrene D, which were also detected in previous Scaligeria EOs studies [38]. In our earlier study on the EOs of the flowering aerial parts of $S$. napiformis, which was collected from two localities in Turkey were analyzed resulting in the characterization of $(E)$ - $\beta$-farnesene, germacrene $\mathrm{D}$, $\alpha$-zingiberine and spathulenol as the main constituents. Overall, 46 compounds were reported from SN-1 and SN-2 aerial part EOs and spathulenol (10.6\%) was identified as the main compound in Scaligeria sp. previously [18]. In another study it was suggested that the distribution of pseudoisoeugenol and propenylphenol derivatives could be used as a taxonomic markers for Pimpinella sp. [41]. In this present study, propenylphenol derivatives and also $(E)$-anethole was found as the major constituent in SL-1, SL-2 and SL-3 oils, respectively. However, S. lazica differed from the other Scaligeria species due to its phytochemical consituents and converged rather to the Pimpinella genus. In addition, geijerene derivatives were identified as the main compounds in the $S$. tripartita oil, which distinguished them from other Scaligeria species on account of this finding. In the other Scaligeria oil analysis, $\delta$-cadinene was characterized as the main component, however only in the SC-1 oil, as seen in Tables 2-4.

Compared to previous studies of the herba and fruit of S. lazica oil collected from Rize-Camlıhemşin analyzed $[35,36]$, our present study on the flowering herba of $S$. lazica collected from three different localities were characterized as different in phytochemical volatile constituents, as seeen in Table 1. The S. triptartita root, fruit, stem and leaf essential oil from Ordu was previously studied reported [17]. In this present study, the flowering herbal parts of $S$. tripartita was collected from Rize and compared to both oil contents as seen in Tables 2 and 4 . In a recent report, S. napiformis flowering herba essential oil chemistry was published by the Baldemir and co-authors [22]. The essential oil of S. meifolia from Turkey were evaluated and samples were collected from two different localities for ssential chemical analysis (Table 1). The chemistry of $S$. meifolia essential oil collected from Iran was reported [39], where the results were different from our present study in terms of main components (Table 2, Table 4). To the best of our knowledge there was no information on the oil analysis both of $S$. hermonis and $S$. glaucescens, which is the first chemo-systematic study among accepted Scaligeria species from Turkey. 
Table 4. Previous reports of Scaligeria essential oils.

\begin{tabular}{|c|c|c|c|}
\hline Plant name & Part used & Main Compounds (\%) & Reference \\
\hline S. tripartita & $\begin{array}{c}\text { stems and leaves } \\
\text { fruit } \\
\text { root }\end{array}$ & $\begin{array}{c}\text { Geijerene (36.8) } \\
\text { (Z)- } \beta \text {-farnesene (9.4) } \\
\beta \text {-Bisabolene (9) } \\
\text { Geijerene (54.6) } \\
\text { Geijerene isomer not } \\
\text { identified (12) } \\
\beta \text {-Bisabolene (7.1) } \\
\text { 4-methoxy-2-(3-methyl- } \\
\text { oxiranyl) phenyl angelate } \\
(36.9) \\
\text { Geijerene }(28.5) \\
\text { Dictamnol (9.4) }\end{array}$ & {$[15]$} \\
\hline S. napiformis A* & aerial parts & $\begin{array}{c}\text { (E)- } \beta \text {-farnesene }(22.2) \\
\text { Germacrene D (19.7) } \\
\alpha \text {-Zingiberene (5) }\end{array}$ & {$[18]$} \\
\hline S. napiformis B** & aerial parts & $\begin{array}{c}\text { Germacrene D (32.7) } \\
\text { Spathulenol (10.6) } \\
\text { (E)- } \beta \text {-farnesene (7.4) }\end{array}$ & \\
\hline S. lazica & aerial parts & $\begin{array}{c}(E) \text {-Anethole }(49.7) \\
\beta \text {-caryophyllene }(19.3) \\
(Z)-\beta \text {-farnesene }(10.2)\end{array}$ & {$[27]$} \\
\hline S. lazica & fruit & $\begin{array}{c}\text { (Z)- } \beta \text {-farnesene }(89.2) \\
(\mathrm{E}) \text {-Anethole }(1.6) \\
\delta \text {-2-carene }(0.9)\end{array}$ & [28] \\
\hline S. assyriaca & $\begin{array}{l}\text { leaf } \\
\text { fruit }\end{array}$ & $\begin{array}{c}\text { Germacrene D (21.1) } \\
\beta \text {-caryophyllene (13.4) } \\
\alpha \text {-copaene (10.2) } \\
\text { Myristicin (24.3) } \\
\text { Germacrene D (13.7) } \\
\text { Elemicine (11) }\end{array}$ & {$[37]$} \\
\hline S. cretica & aerial parts & $\begin{array}{c}\text { Sabinene (13.7) } \\
\beta \text {-farnesene (29.7) } \\
\text { Germacrene D (28.4) }\end{array}$ & {$[38]$} \\
\hline S. meifolia & aerial parts & $\begin{array}{c}\text { Germacrene D (24.2) } \\
\text { Germacrene B (14.8) } \\
\text { Limonene (14.2) }\end{array}$ & [39] \\
\hline
\end{tabular}

According to literature, EO and their constituents display a broad antibacterial activity spectrum, and could therefore be used in pharmaceutical industries against microbial resistance versus conventional antibiotics $[1,3-4,8]$. In this present study, according to the in vitro antibacterial results the tested oils except for ST-1 showed moderate antimicrobial activity but also SH-1 oil was only effective oil sample against $B$. cereus. SC-1 oil was more effective against Gram positive bacteria compared to other Scaligeria samples (Table 3). Possibly the antimicrobial effects of SL-1, SL-2 and SL-3 oils can be linked to the main component $(E)$-anethole (Figure 1). Also, this compound was used as chemical precursor of paramethoxyamphetamine in the clandestine manufacture of amphetamines [47]. It is well known that anethole is therapeutically an important compound, which was reported to have strong anti-inflammatory, antifungal, antibacterial, and antiplatelet activities among others [4853]. 


\section{Conclusion}

Overall, GC-FID and GC-MS profiles of the investigated Scaligeria taxa exhibited important differences between the two taxa (S. lazica and S. tripartita) and other Scaligeria taxa (S. napiformis, $S$. meifolia, $S$. capillifolia, $S$. hermonis, $S$. glaucescens) highlighting the existence of different main chemical constituents. Thus, the results of this study certainly contributed to the taxonomy of the genus Scaligeria via essential oil chemistry. On the other hand, while germacrene D was determined as main component in the other Scaligeria species it can be accepted mainly to the discrimination on the Scaligeria genus, due to characteristic chemical compositions $S$. lazica and S. tripartita's placement in Scaligeria is questionable. The status of Scaligeria taxa may become more evident, when other targeted studies such as on molecular, chemical analysis of the root and fruit, anatomical and morphological levels are completed.

\section{Acknowledgments}

This work was supported by Research Fund of the Erciyes University Research Fund, Kayseri, Turkey [Project Number: TOA-2014-4557]. Authors are grateful to Assist. Prof. Metin Armağan of Yüzüncü Y1l University, Van, Turkey for collection and identification of S. glaucescens.

\section{Supporting Information}

Supporting information accompanies this paper on http://www.acgpubs.org/RNP

\section{ORCID}

Ayşe Baldemir: 0000-0003-2473-4837

Betül Demirci: 0000-0003-2343-746X

Mehmet Yavuz Paksoy: 0000-0001-9581-4514

Selen İlgün: 0000-0002-8544-0683

Müberra Koşar: 0000-0002-1559-998X

Kemal Hüsnü Can Başer: 0000-0003-2710-0231

Fatih Demirci: 0000-0003-1497-3017

\section{References}

[1] A.E. Edris (2007). Pharmaceutical and therapeutic potentials of essential oils and their individual volatile constituents: A review, Phytother. Res. 21, 308-323.

[2] S.L. Crockett (2010). Essential oil and volatile components of the genus Hypericum (Hypericaceae), Nat. Prod. Commun. 5, 1493.

[3] G. Lang and G. Buchbauer (2012). A review on recent research results (2008-2010) on essential oils as antimicrobials and antifungals: A review, Flav. Frag. J. 27, 13-39.

[4] B. Teixeira, A. Marques, C. Ramos, N.R. Neng, J.M. Nogueira, J.A. Saraiva and M.L. Nunes (2013). Chemical composition and antibacterial and antioxidant properties of commercial essential oils, Ind. Crops Prod. 43, 587-595.

[5] S.D. Cox, C.M. Mann, J.L. Markham, H.C. Bell, J.E. Gustafson, J.R. Warmington and S.G. Wyllie (2000). The mode of antimicrobial action of the essential oil of Melaleuca alternifolia (tea tree oil), J. Appl. Microbiol. 88, 170-175.

[6] R.J.W. Lambert, P.N. Skandamis, P.J. Coote and G.J. Nychas (2001). A study of the minimum inhibitory concentration and mode of action of Oregano essential oil, thymol and carvacrol, J. Appl. Microbiol. 91, 453-462.

[7] S. Burt (2004). Essential oils: their antibacterial properties and potential applications in foods-a review, Int. J. Food Microbiol. 94, 223-253.

[8] J.S. Raut and S.M. Karuppayil (2014). A status review on the medicinal properties of essential oils, Ind. Crops Prod. 62, 250-264. 
[9] E. Nemeth and G. Szekely (2000). Floral biology of medicinal plants I. Apiaceae species, J. Am. Soc. Hortic. Sci. 6, 133-136.

[10] J.T. Knudsen, R. Eriksson, J. Gershenzon and B. Stahl (2006). Diversity and distribution of floral scent, Bot. Rev. 72, 1-120.

[11] F. Oroojalian, R. Kasra-Kermanshahi, M. Azizi and M.R. Bassami (2010). Phytochemical composition of the essential oils from three Apiaceae species and their antibacterial effects on food-borne pathogens, Food Chem. 120, 765-770.

[12] M. Vigan (2010). Essential oils: renewal of interest and toxicity, Eur. J. Dermatol. 20, 685-692.

[13] K.A. Hammer and C.F. Carson (2011). Antibacterial and antifungal activities of essential oils. In: Lipids and Essential Oils as Antimicrobial Agents, ed: Thormar Halldor, John Wiley \& Sons, Ltd, UK, pp. 255-306.

[14] K.H.C. Baser and N. Kirimer (2014). Essential oils of Anatolian Apiaceae - A Profile, Nat. Vol. Essent. Oils 1, 1-50.

[15] P.F. Stevens (1972). Scaligeria DC., In: Flora of Turkey and The East Aegean Islands. ed: P.H. Davis, Edinburgh University Press, Edinburgh, Vol. 4, pp. 333-336.

[16] A. Güner, B. Akyıldırım, M.F. Alkayış, B. Çıngay, S.S. Kanoğlu, A.M. Özkan, M. Öztekin, G.N. Tuğ (2012). Türkçe bitki adları, In: Türkiye Bitkileri Listesi (Damarlı Bitkiler). eds: A. Güner, S. Aslan, T. Ekim, M. Vural, M.T. Babaç, Nezahat Gökyiğit Botanik Bahçesi ve Flora Araştırmaları Derneği Yayını, İstanbul, 77.

[17] N. Tabanca, B. Demirci, K.H.C. Baser, E. Mincsovics, S.I. Khan, M.R. Jacob and D.E. Wedge (2007). Characterization of volatile constituents of Scaligeria tripartita and studies on the antifungal activity against phytopathogenic fungi, J. Chromatogr. Sci. B, 850, 221-229.

[18] F.W. McLafferty and D.B. Stauffer (1989). The Wiley/NBS Registry of Mass Spectral Data, New York: $\mathrm{J}$ Wiley and Sons.

[19] W.A. Koenig, D. Joulain and D.H. Hochmuth (2004). Terpenoids and Related Constituents of Essential Oils, Hamburg, Germany: MassFinder 3.

[20] D. Joulain and W.A. Koenig (1998). The Atlas of Spectra Data of Sesquiterpene Hydrocarbons, Hamburg: EB-Verlag.

[21] ESO 2000 (1999). The Complete Database of Essential Oils, The Netherlands: Boelens Aroma Chemical Information Service.

[22] A. Baldemir, B. Demirci, S. Ilgun, M. Kosar and M.Y. Paksoy (2016). Essential oil composition of Scaligeria napiformis native to Turkey, Chem. Nat. Compd. 52, 1100-1101.

[23] CLSI (NCCLS) M27-A2 (2006). Performance standards for antimicrobial disk susceptibility tests; Approved Standard, Seventh Edition.

[24] CLSI (NCCLS) M100-S17 (2007). Performance standards for antimicrobial susceptibility testing; Approved Standard, Seventeenth Edition.

[25] H.E. Temel, B. Demirci, F. Demirci, F. Celep, A. Kahraman, M. Doğan and K.H.C. Başer (2016). Chemical characterization and anticholinesterase effects of essential oils derived from Salvia species, J. Essent. Oil Res. 28, 322-331.

[26] V.I. Babushok, P.J. Linstrom and I.G. Zenkevich (2011). Retention Indices for Frequently Reported Compounds of Plant Essential Oils, J. Phys. Chem. Ref. Data 40, 1-47.

[27] E.M. Gaydou, R. Faure, J.-P. Bianchini, G. Lamaty, O. Rakotonirainy and R. Randriamiharisoa (1989). Sesquiterpene composition of basil oil. Assignment of the ${ }^{1} \mathrm{H}$ and ${ }^{13} \mathrm{C}$ NMR spectra of $\beta$-elemene with two-dimensional NMR, J. Agric. Food Chem. 37, 1032-1037.

[28] H. Noorizadeh, A. Farmany and M. Noorizadeh (2011). Application of GA-PLS and GA-KPLS calculations for the prediction of the retention indices of essential oils, Quim. Nova. 34, 1398-1404.

[29] T. Erdoğan, B. Sümer, Ö. Özçınar, U. Çakıılıığlu, B. Demirci, K. H. C. Başer and B. Kıvçak (2017). Essential Oil Composition of Three Centaurea Species from Turkey: Centaurea aggregata Fisch. \& Mey. ex. DC. subsp. aggregata, C. balsamita Lam. and C. behen L., Rec. Nat. Prod, 1, 69-73.

[30] Y. Frum and A.M. Viljoen (2006). In vitro 5-lipoxygenase and anti-oxidant activities of South African medicinal plants commonly used topically for skin diseases, Skin Pharmacol. Physiol. 19, 329-335.

[31] H. Noorizadeh and A. Farmany (2010). Exploration of linear and nonlinear modeling techniques to predict of retention index of essential oils, J. Chin. Chem. Soc. 57, 1268-1277.

[32] B.T. Sullivan (2002). Evidence for a sex pheromone in bark beetle parasitoid Roptrocerus xylophagorum, J. Chem. Ecol. 28, 1045-1063.

[33] F. Bakkali, S. Averbeck, D. Averbeck and M. Idaomar (2008). Biological effects of essential oilsA Review, Food Chem.Toxicol. 46, 446-475. 
[34] S. Jaric, M. Mitrovic, L. Djurdjevic, O. Kostic and G. Gajic (2011). Phytotherapy in medieval Serbian medicine according to the pharmacological manuscripts of the Chilandar Medical codex (15-16th centuries), J. Ethnopharmacol. 137, 601-619.

[35] K.H.C. Başer, T. Özek and M. Kürkçüoğlu (1993).The Essential oil of Scaligeria lazica Boiss., J. Essent. Oil Res. 5, 463-464.

[36] K.H.C. Başer, T. Özek and M. Kürkçüoğlu and A. Güner (1995). The Essential oil of Scaligeria lazica Boiss., J. Essent. Oil Res. 7, 557-58.

[37] M.N. Navaeil and M. Mirza (2009). Chemical composition of the essential oils from fruits and leaves of Scaligeria assyriaca Freyn \& Bornm, Iran J. Med. Aromatic Plants 25, 398-402.

[38] E. Evergetis, A. Michaelakis and S.A. Haroutounian (2012). Essential oils of Umbelliferae (Apiaceae) family taxa as emerging potent agents for mosquito control. Integrated pest management and pest control-current and future tactics, InTech. 613-638.

[39] V. Rowshan and A. Tarakemeh (2013). Chemical composition of Scaligeria meifolia Boiss. essential oil from Iran, Herba Pol. 59, 29-34.

[40] N. Tabanca, A.W. Douglas, E. Bedir, F.E. Dayan, N. Kırımer, K.H.C. Başer, Z. Aytaç, I.A. Khan and B.E. Scheffler (2005). Patterns of Essential oil relationship in Pimpinella (Umbelliferae) based on phylogenetic relationships using nuclear and chloroplast sequences, Plant Genet. Resour. 3, 149-169.

[41] N. Tabanca, B. Demirci, T. Ozek, N. Kirimer, K.H.C. Baser, E. Bedir, I.A. Khan and D.E. Wedgea (2006). Gas chromatographic-mass spectrometric analysis of essential oils from Pimpinella species gathered from Central and Northern Turkey, J. Chromatogr. A, 1117, 194-205.

[42] A. Orav, A. Raal and E. Arak (2008). Essential oil composition of Pimpinella anisum L. fruits from various European countries, Nat. Prod. Res. 22, 227-232.

[43] P.M. Santos, A.C. Figueiredo, M.M. Oliveira, J. Barroso, L.G. Pedro, S.G. Deans, A.K.M. Younus and J.J.C. Scheffer (1998). Essential oils from hairy root cultures and from fruits and roots of Pimpinella anisum, Phytochem. 48, 455-460.

[44] J.J. Brophy, R.J. Goldsack, C.J. Fookes and I. Hutton (2004). Leaf oils of the endemic Melicope (Rutaceae) of Lord Howe Island, J. Essent. Oil Res. 16, 449-452.

[45] J.A. John, J.O. Jose, V. George, N.S. Pradeep and M.G. Sethuraman (2008). Volatile Constituents and Antibacterial Activity of Leaf Oil of Thottea ponmudiana Sivar, J. Essent. Oil Res. 20, 460-463.

[46] C. Kossouoh, M. Moudachirou, V. Adjakidje, J.C. Chalchat, G. Figuérédo and P. Chalard (2011). Volatile constituents of Chromolaena odorata (L.) RM King \& H. Rob. leaves from Benin, J. Essent. Oil Bear. Pl. 14, 224-228.

[47] D. Waumans, N. Bruneela and J. Tytgat (2003). Anise oil as para-methoxyamphetamine (PMA) precursor, Forensic Sci. Int. 133,159-70.

[48] M. De, A.K. De, P. Sen and A.B. Banerjee (2002). Antimicrobial properties of star anise (Illicium verum Hook f), Phytother. Res. 16, 94-95.

[49] K.I. Fujita, T. Fujita and I. Kubo (2007). Anethole, a potential antimicrobial synergist, converts a fungistatic dodecanol to a fungicidal agent, Phytother. Res. 21, 47-51.

[50] F. Senatore, F. Oliviero, E. Scandolera, O. Taglialatela-Scafati, G. Roscigno, M. Zaccardelli and E. De Falco (2013). Chemical composition, antimicrobial and antioxidant activities of anethole-rich oil from leaves of selected varieties of fennel [Foeniculum vulgare Mill. ssp. vulgare var. azoricum (Mill.) Thell], Fitoterapia 90, 214-219.

[51] I. Kubo, K. Fujita and K. Nihei (2008). Antimicrobial activity of anethole and related compounds from aniseed, J. Sci. Food Agric. 88, 242-247.

[52] C.H. Chen and L.A. deGraffenried (2012). Anethole suppressed cell survival and induced apoptosis in human breast cancer cells independent of estrogen receptor status, Phytomedicine 19, 763-767.

[53] M. Tognolini, V. Ballabeni, S.Bertoni, R.Bruni, M. Impicciatore and E. Barocelli (2007). Protective effect of Foeniculum vulgare essential oil and anethole in an experimental model of thrombosis, Pharmacol. Res. 56, 254-260.

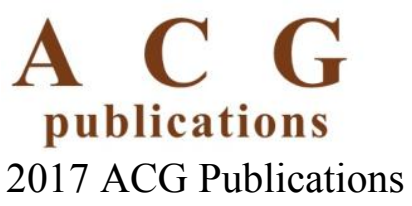

